\title{
Calcification of Axial Skeleton and Segmentation Muscular Regeneration of Gecko's Tail (Gekko Gecko Linnaeus, 1758)
}

\author{
Azkiya Zahrotus Syarifah ${ }^{1 *}$, Muhammad Ja'far Luthfi', Alfiatun Wa'is Khusnul Khatimah ${ }^{2}$, Aulya Nidaur \\ Rahmah $^{3}$ \\ ${ }^{1}$ Madrasah Aliyah Kartayuda Jl Pasar No 10 Wado Kedungtuban Blora Tel. (0296) 4270453 NPSN: 20364946 \\ ${ }^{2}$ Biology Education Department, ${ }^{3}$ Biology Department, Faculty of Science and Technology, UIN Sunan Kalijaga \\ Jl. Marsda Adisucipto No 1 Yogyakarta 55281, Indonesia. Tel. +62-274-540971, Fax. +62-274-519739 \\ ${ }^{1}$ Email: azky_imutzh@yahoo.co.id
}

\begin{abstract}
Gecko is an animal that can carry out autotomy. Research on gecko tail autotomy has been carried out, but there are still few who research the axial skeleton that focuses on vertebrae caudales and segmentation muscular arranged, this is the background of this research. This research is expected to be data base further research and as a comparison between animals that can induce further autotomy. This study aims to determine the macrostructure and microstructure of axial axial gecko tail regenerates and determine the anatomical microstructure of muscular regeneration of gecko tail. The methods used were X-Ray, Alizarin Red S and Alcian Blue, Paraffin method with hematoxylin-eosin staining, and Mallory Triple Stain. The results showed that the color of the gecko tail regenerate was paler compared to the original tail. On observations using radiological rays and alizarin staining showed that the original tail would look segmented and have a process. The original gecko tail is composed of bones, because it is red which shows perfectly calcified bone. While the gecko tail regenerate is composed of cartilage that is shaped like a long pipe glazed red because it has calcified. At the end of the tail there is also a blue color, this indicates that the gecko tail regenerate has not been completely calcified. Segmentation Muscular of the original gecko tail, when viewed longitudinally, indicates a segment that extends from one process to the skin and when viewed from the cross has only four muscle segments separated by the septum. While the gecko tail regenerate when viewed in a longitudinal manner there is no segment and when seen transversely there are 12 muscle segments seen. Muscles are composed of a collection of myotubes that form myotomes, each myotomes limited by myoseptum.
\end{abstract}

Keywords: Axial skeleton calcification, Gecko, Regenerate tail, Segmentance muscular

\section{INTRODUCTION}

Life of each organism is influenced by both abiotic and biotic interactions, both directly and indirectly. Most interactions are related to food competition, eating or avoiding being eaten. To avoid the enemy there are several methods used, namely by camouflage, hiding, escaping, and autotomy (Kimball, 1983; Halliday, 1986).

A tail autotomy is the ability to cut off some limbs as a way to save themselves from predators. Some Lacertilia members such as Mabouya multifasciata Kuhl and Hemidactylus sp have the ability to tail autotomy when threatened or captured by their enemies, namely by severing part or all of their tail. This animal will use the opportunity to escape when the tail is released. This loose tail movement will attract the attention of the enemy (Bustard, 1968: Bellair and Bryant, 1986; vitt, 1981; Storer et al., 1979).

Gecko is an animal that can do autotomy. This was evidenced in an experiment conducted by Woodlan (1920) which stated that more than $50 \%$ of geckos carry out tail autotomy, but this study does not state the ability of the gecko autotomy itself, nor does it state how the macro structure and microstructure vertebral caudal and muscle segment of the tail geckos both original and regenerated. In addition, there are still a few who are researching the axial skeleton which focuses on vertebral caudales and segmentation muscular arranged, which is the background of the compilation of this research. This research is expected to become the next research data base (Lutfi, 2002).

\section{MATERIALS AND METHODS}

The materials used in this study were: $24-2$ years old gecko tillers as many as 24 were obtained from the PASTY animal market in Bantul Regency, Hongkong caterpillar as gecko food, Alcohol, Mallory Triple Stain, Alizarin Red S, Alcian Blue, $\mathrm{KOH}$, Acetone, Glycerin, Chloroform, Glacial Acetic Acid, Hematokxylin, Eosin, Bouin, Paraffin, Xylol, Aquades, Toluene, and Entellan.

The tools used in this study are cages equipped with water and food containers, $X$-rays, surgical instruments, microscopes, paraffin ovens, microtomes, glass alarms, staining jar, flacon bottles, slides, glass covers, tissue bags, cotton, petri dishes, slide warmers, gauze, tubes, tube plugs, and threads.

To find out whether is occurring, vertebrae caudales macroscopic tail regeneration of observation is carried out:

a. by observing the presence or absence of reinforcement on intact regenerate tail preparations from results $X$-ray. 
b. Microscopic, namely by observing the histocirnia images withstaining Hematoxylin-Eosin and Mallory Triple Stain.

To find out the order of the process of muscular tail regeneration, it was observed in intact preparations and slices of tail regenerate preparations made longitudinally with staining Hematoxylin-Eosin and Mallory Triple Stain.

\section{RESULTS AND DISCUSSION}

\section{Observation Results}

Observation of Macro Anatomical Structure by X-ray Method

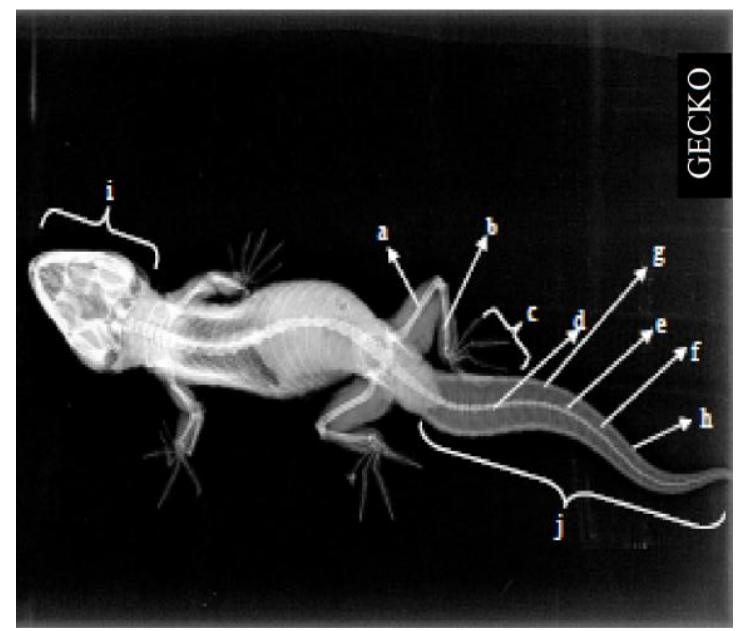

Figure 1. Axial axle of original gecko tail with $X$-ray, using machine $X$ ray from dorsal longitudinal section with description: (a) Femur, (b) Tibia-fibula, (c) Tarsal-metatarsal-phalanges/digiti, (d) Centrum, (e) Processus Transversal, (f) Fat, (g) Tissue muscle (h) Tissue skin (i) Thorakales tissue (j) Caudales.

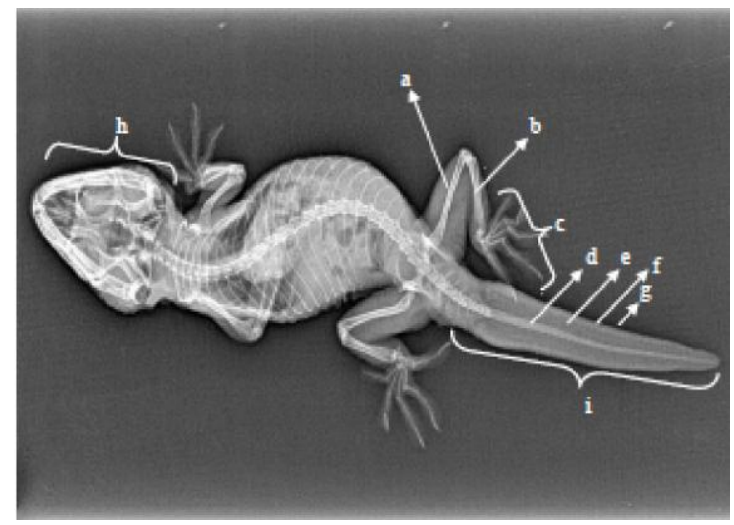

Figure 2. Axial axle of gecko tail regenerate with $X$-ray longitudinal cross section of dorsal with description: (a) Femur, (b) Tibia-fibula, (c) Tarsal-metatarsal-phalang/digiti, (d) Tube carilago, (e) Fat, (f) Tissue muscle, (g) Tissue skin (h) Thorakales tissue (i) Caudales.
Observation of intact preparations by Staining Method Alizarin Red S and Alcian Blue

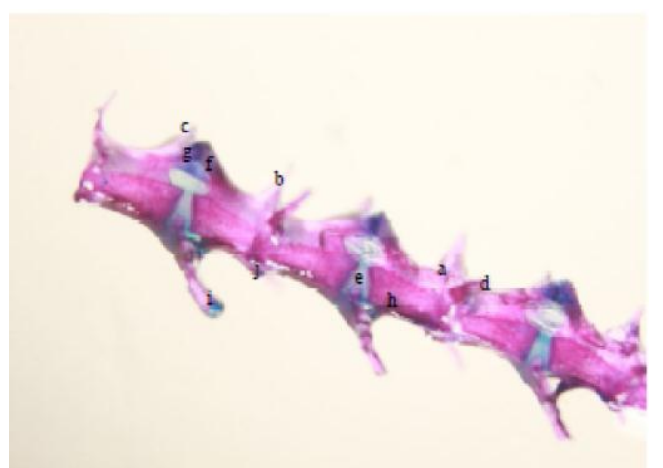

Figure 3. Axial axle of original gecko tail with Alizarin Red $S$ and Alcian Blue, longitudinal cross section from the lateral, with description: (a) Square autotomy, (b) Processus of the dorsal/anterior neuralbifida, (c) Spinaneuralposterior, (d) Processustransverse, (e) VertebralInter, (f) Prezygapophysis, (g) Postzygapophysis, (h) Centrum, (i) Chevron, (j) Processus ventral.

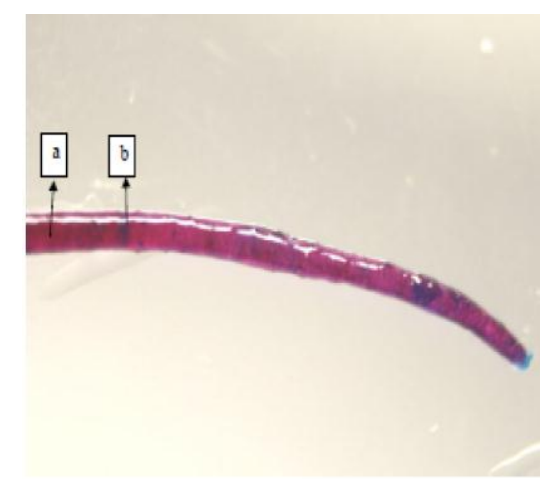

Figure 4. Axial Skeleton regenerate tail gecko with Alizarin Red $S$ and Alcian Blue longitudinal cross-section of the lateral ends, $6 \times 1$ magnification, with the caption: (a) Calcified cartilage tube, (b) Uncalified cartilage tube.

\section{Observation of Gecko Anatomy Microstructure} Making Sliced Preparations using Hematoxylin and Eosin Staining Method

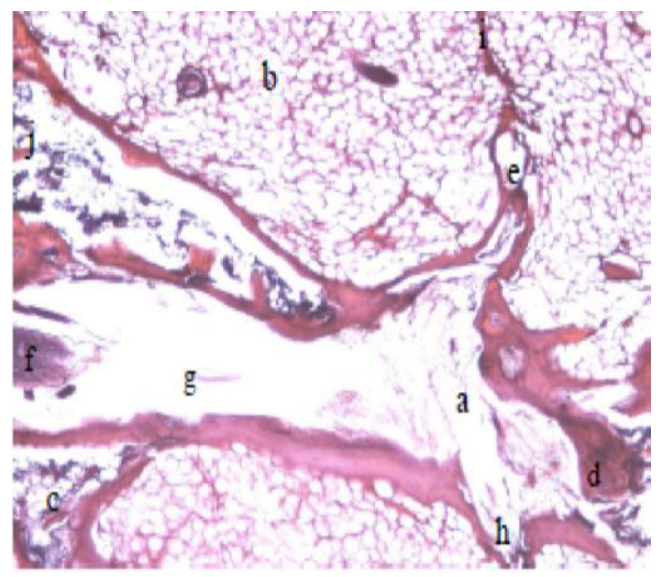

Figure 5. Original gecko tail bone, research/light microscope, longitudinal cross section,staining Hematoxylin-Eosin, 4x10 magnification, with description : (a) Plan autotomy, (b) Perivertebral fat tissue, (c) Centrum vertebrae, (d) Transverse Processus, (e) Dorsal processus/anterior neural spina, (f) Spinal Medulla, (g) Vertebral canal, (h) Ventral Processus, (i) Autotomy segment, (j) Posterior. 


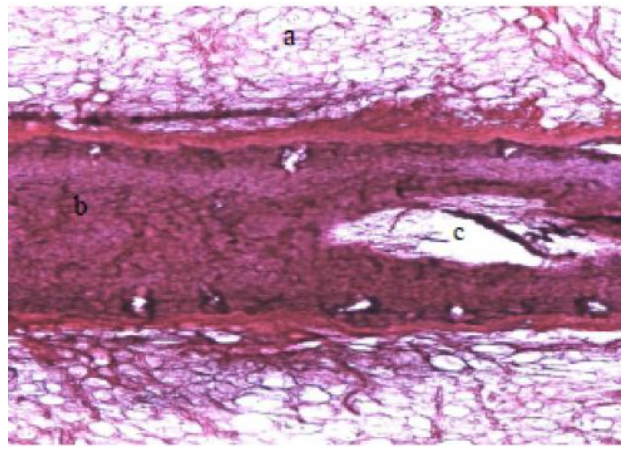

Figure 6. Bone gecko tail regenerate, staining Hematoxylin-Eosin, research/light microscope, longitudinal cross section, 10x10 magnification, with description: (a) Fat tissue, (b) Cartilage tube, (c) Ependymocyti.

\section{Observation of Micro Anatomy with Segmentation of Muscular Using Staining Methods Hematoxylin and Eosin}

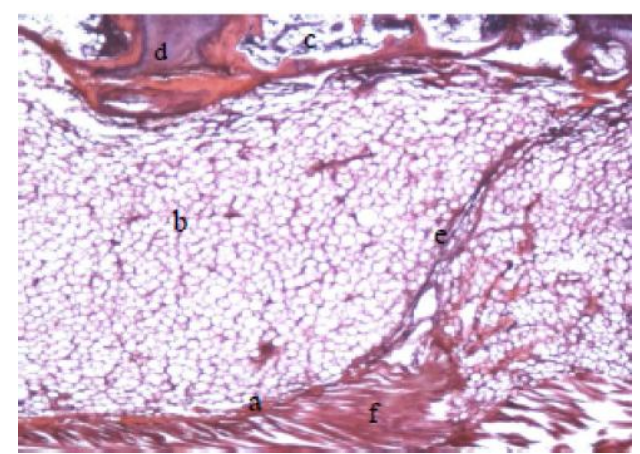

Figure 7. Segmentation Muscular of original gecko tail, staining Hematoxylin-Eosin, research/light microscope, longitudinal cross section, 4x10 magnification, with description: (a) Autotomy Muscle Segments (b) Fat Tissue, (c) Centrum vertebrae, (d) Intervertebrae, (e) Autotomy, (f) Muscular.

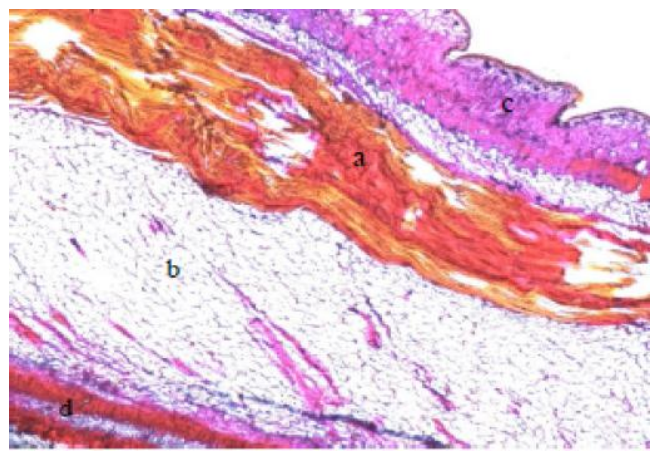

Figure 8. Segment Gecko regenerate tail color Mallory Triple Stain, research/light microscope, gecko tail bone longitudinal cross section, 4x10 magnification, with description: (a) Muscle, (b) Fat tissue, (c) Skin, (d) Tube cartilage.

\section{Discussion}

Observation using $X$-rays (Figure 1) is clearly seen vertebrae gecko both from the piston to the caudal. The original gecko tail shows the existence of a transverse process composed of bone segments that have a plain autotomy.
In contrast to the original gecko tail, the gecko tail regenerate (Figure 2) does not have segmented bones because it only has a tubular bone that extends posteriorly. Muscles that appear to be different from the original tail have muscles that are segmented or segmented as well, while the muscles in the regeneration of the tail are innocent without any separator that separates them. The skin that appears on the gecko tail is also different, the regenerate looks plain while the skin on the original gecko tail is jagged.

In making the whole preparation using Alizarin Red S-Alcian Blue coloring, that the tail of the regenerate is different from the original tail. By using this coloring the bone will be red, while the cartilage will blue. This is because Alizarin Red S-Alcian Blue is a differential dye that will be able to distinguish between bone tissue and cartilage.

The original gecko tail coloring with Alizarin Red S-Alcian Blue from the lateral (Figure 3) clearly shows the plain autotomy stretches from the ventral to the dorsal/anterior neural spine process. In parts of this bone segment are seen centrum, the, intervertebrae, and chevron as supporting the tail section bottom and the transverse process that is next to the plain autotomy. Even from the lateral bone perfectly smooth in red, this is because bone is composed of calcium.

According to Lutfi (2002), the calcification process (reinforcement) or ossification will cause the cartilage pipe to be red. The red color is caused by the bonding of the dye Alizarin Red Celcius Blue with calcium ions. If the color is more red (dark red), the more calcium ions in the cartilage tube and indicate that the calcification process is more perfect. Regenerate the old gecko tail on the cartilage looks red. The red color in the cartilage tube shows that calcification has occurred. Regenerate a young gecko tail, it will be blue because it has not experienced calcification. This is shown in the end cartilage tube (Figure 4) which has not been completely calcified.

Observation of gecko micro anatomy, observations on longitudinal muscle slice preparations on the original gecko tail with hematoxylin-eosin staining (Figure 5) showed that the original tail muscle had an autotomy segment in order to facilitate the autotomy process and in Mallory triple stain (Figure 6) showed that regenate tail muscle does not have an autotomy segment, the muscle is also plain.

\section{CONCLUSIONS}

Based on the results of observations and data analysis and the discussions that have been conducted, the following conclusions are obtained:

1. The color of the regenerate tail is paler compared to the original tail. On the tail of the original gecko when viewed using radiological rays and staining Alizarin of the original tail will look segmented and have a processus. The original gecko tail is composed of bones, because it is red which shows 
perfectly calcified bone. While the gecko tail regenerate is composed of cartilage that is shaped like a long pipe glazed red because it has calcified and also the tip of the tail is blue, this indicates that the gecko tail regenerate has not been completely calcified.

2. The segment of the original gecko tail muscle when viewed longitudinally is a segment that extends from one process to the coolie and when viewed from the cross has only four muscle segments separated by the septum. While the gecko tail regenerate when viewed in a longitudinal manner there is no segment and when seen transversely there are 12 muscle segments seen. Muscles are composed of a collection of myotubes that form myotomes, each myotomes limited by myoseptum.

\section{ACKNOWLEDGMENTS}

The author would like to thank to the management of apprenticeship program of faculty of science and technology for guidance during the research, to the management of biology, medicine and natural product chemistry \& kaunia journal for assistance in paper writing.

\section{REFERENCES}

Andayani, MML. 2007. Lizard Tail Microanatomy (Mabouya multifasciata Kuhl) Wound Healing Phase After Autotomy and Gamma Ray Irradiation. Description of Gadjah Mada University (Not Published).

Anonymous. 1985. Biology of the Reptilia (vol.15). New York: A Wiley-interscience publication.

Bellairs, ADA And SV Bryant. 1985. Autotomy and Regeneration in Reptiles. In Biology of the Reptilia. (C. Gans, F. Billet, eds) vol. 15 Development B. Academic Press. London. Hal 301-410.

Carlson, BM. 1988. Regeneration, in the Encylopedia Americana. International Edition. Vol 32. Grolier inc. Coneticut. 340.

Gardner, Gray, and O'Rahilly. 1995. Anatomy (Study of the Human Body). Translated by ZS Bustami. University of Indonesia Publisher, Jakarta.

Halliday, TR and Adler, K. 1986. The Encyclopedia of Reptiles and Amphibian. Andromeda Oxford Ltd. England. Pp. 63-64.

Kimball, JW. 1983. Biology. Fifth edition. Jakarta: Erlangga.

Lutfi, M. Jafar. 2002. Calcification of the Axial Skeleton and Autotomy Capability of Lizard Tails (Mabouya multifasciata kuhl). Thesis. Gajah Mada University (Not Published).

Soesilo, NP 1999. Role of Ependima Layer in Regeneration of Lizard Tails (Mabouya multifasciata Kuhl), Biology, Vol. 2, No.8: 419-450.

Woodland, WNF. 1920. Some Observations on Caudal Autotomy and Regeneration in Gecko (Hemydactylus flaviridis Ruppel), with notes on the tail of Sphenodon and Pygopus. Quaterly juornal of Microscopical science. 65; 63-100.

Weichert, C.K. 1970. Anatomy of The Chordates Fourth Edition. Tokyo: Mc Graw hill book company. Hal 34-40. 